\title{
CHARACTERIZATION OF TILT AND TWIST LOW ANGLE GRAIN BOUNDARIES AND THEIR EFFECTS ON INTERMEDIATE-TEMPERATURE CREEP DEFORMATION BEHAVIOUR
}

\author{
Yao Wang ${ }^{1}$, Dong Wang ${ }^{1}$, Gong Zhang ${ }^{1}$, Langhong Lou ${ }^{1}$, Jian Zhang ${ }^{1,2}$ \\ ${ }^{1}$ Superalloys Division, ${ }^{2}$ Shenyang National Laboratory for Materials Science, Institute of Metal Research, Chinese Academy of Sciences, \\ 72 Wenhua Road, Shenyang 110016, China
}

Keywords: Low angle grain boundary, Creep deformation, Single crystal Ni-based superalloy, Dislocation

\begin{abstract}
The characterization of tilt and twist low angle grain boundaries (LAGBs) has been performed, and the influences of LAGBs on intermediate-temperature creep deformation behavior have been investigated in a single crystal Ni-based superalloy. It is found that the tilt boundary is composed of intrinsic edge dislocations, and the twist boundary mainly consists of intrinsic screw dislocations. LAGB type is found to have a significant influence on the rupture life of SX superalloy deformed at $760{ }^{\circ} \mathrm{C}$. The tilt boundary shows a much better creep resistance compared to that of a twist boundary. The dislocation configuration near the grain boundary indicates that most of the mobile dislocations can pass through the tilt boundary, but they are basically inhibited and piled-up at the twist boundary during creep deformation.
\end{abstract}

\section{Introduction}

Single crystal (SX) Ni-based superalloys are widely used as blade materials in advanced aeroengines and industrial gas turbines (IGTs) because of their excellent mechanical properties. However, when casting these SX components especially large-size IGT blades, there is a high probability of inducing LAGBs due to the intersection or distortion of primary dendrites during directional solidification $[1,2]$. An elastically distorted region is generated around LAGBs, and the resulting local strain field interacts with the moving dislocations during deformation, which may lead to premature damage.

Experimental evidence in previous works have demonstrated the detrimental effects of LAGBs in SX superalloys. For example, the creep rupture life of RR2072 SX superalloy with a transverse LAGB at $950{ }^{\circ} \mathrm{C} / 210 \mathrm{MPa}$ and $950{ }^{\circ} \mathrm{C} / 290 \mathrm{MPa}$ decreases exponentially with increasing grain boundary angle from 3 deg to $18 \mathrm{deg}$ [3]. Stinville et al [4] found a steady decrease in creep rupture life and creep strain at rupture with the increasing misorientation angle for René N4 bicrystal superalloy at 982 ${ }^{\circ} \mathrm{C} / 207 \mathrm{MPa}$. Zhao et al [5] also found that once the degree of LAGB is more than $12 \mathrm{deg}$, the instantaneous creep damage of DD6 SX superalloy along LAGB is observed at $800^{\circ} \mathrm{C} / 700 \mathrm{MPa}$. Therefore, LAGB allowance is generally set in casting practice to guarantee the reliability of the SX blades.

The LAGBs are divided into tilt and twist LAGBs according to the relationship between the rotation axes of neighboring crystal lattices and grain boundary interface. This leads to different dislocation configurations at LAGB, i.e., edge dislocations at tilttype and screw dislocations at twist-type LAGBs, respectively. The results of computational simulations show that the occurrence probabilities of these boundary types for the cubic crystal symmetry is uneven [6], with $3.9 \%$ of random boundaries neartwist character and $84.0 \%$ of random boundaries near-tilt character. However, most of the above statements are based on theoretical analysis or experimental observations on simple systems. There is little information in the literature reporting detailed characterization on grain boundary features in complex alloys such as Ni-based superalloys. It is therefore important to further characterize the LAGB and explore the effect of tilt or twist LAGBs on the mechanical behavior and damage mechanisms of the SX superalloys.

Unfortunately it is difficult to observe LAGBs by transmission electron microscope (TEM), especially for the twist LAGB because of its unique structure composed of screw dislocation. It is also difficult to separate the effects of tilt and twist boundaries during creep.

In the present study, the creep behavior of a Ni-based SX superalloy with transverse tilt or twist LAGB at intermediatetemperature was investigated. The interaction mechanisms between mobile dislocations and LAGBs were characterized, and the creep damage mechanisms of SX superalloys with tilt or twist LAGBs were also discussed.

\section{Material and experimental procedures}

DD10 (a 1st generation hot corrosion resistant SX Ni-base superalloy) used in the present experiments contained $12.5 \mathrm{wt} \%$ Cr, 4.5 wt $\%$ Co, 4.0 wt $\%$ Ti, 4.0 wt $\%$ Al, 5.5 wt $\%$ Ta, 5.5 wt $\%$ W, $0.4 \mathrm{wt} \%$ Mo with Ni bal.. Bicrystals with different LAGBs (5 deg, $10 \mathrm{deg}$ and $15 \mathrm{deg}$ ) were grown using the seed crystal method, and a high rate solidification process was used to cast the bicrystals with the size of $200 \mathrm{~mm}$ (length) $\times 80 \mathrm{~mm}$ (width) $\times 20 \mathrm{~mm}$ (thickness). Figure 1 is schematic of bicrystal slab casting prepared by the seed crystal method.

For tilt LAGBs, the seed crystal pairs were obtained from the identical SX bar with [001] orientation. The [001] direction of two seed crystals were both parallel to the solidification direction (case 1 in Figure 1). While fixing the [010] direction of one seed crystal parallel to the width direction of the slab, the other seed was rotated around its [001] axis to achieve the desired bicrystal boundary misorientation $\theta$. For twist LAGBs, the seed crystal pairs were also obtained from the identical SX bar with [001] orientation but both with orientation deviation $\delta / 2$ to [001] direction. The [010] direction of two seed crystals were both parallel to the width direction of slab (case 2 in Figure 1). The two seeds were carefully arranged to achieve the misorientation $\delta$ between the two [001] direction, while keeping the two [010] directions parallel.

The ceramic mold fixed on a copper chill plate was preheated to $1500{ }^{\circ} \mathrm{C}$ before casting. The position of the chill plate was adjusted carefully to achieve the melting of the upper section of the seeds. A withdrawal rate of $3 \mathrm{~mm} / \mathrm{min}$ was used during 
directional solidification. The bicrystal slabs were solution treated at $1250{ }^{\circ} \mathrm{C}$ for $3 \mathrm{~h}$ in air, precipitation heat treated at $1100{ }^{\circ} \mathrm{C}$ for 5 $\mathrm{h}$, and aged at $870^{\circ} \mathrm{C}$ for $24 \mathrm{~h}$, before being cut into specimens for microstructural observation and creep tests.

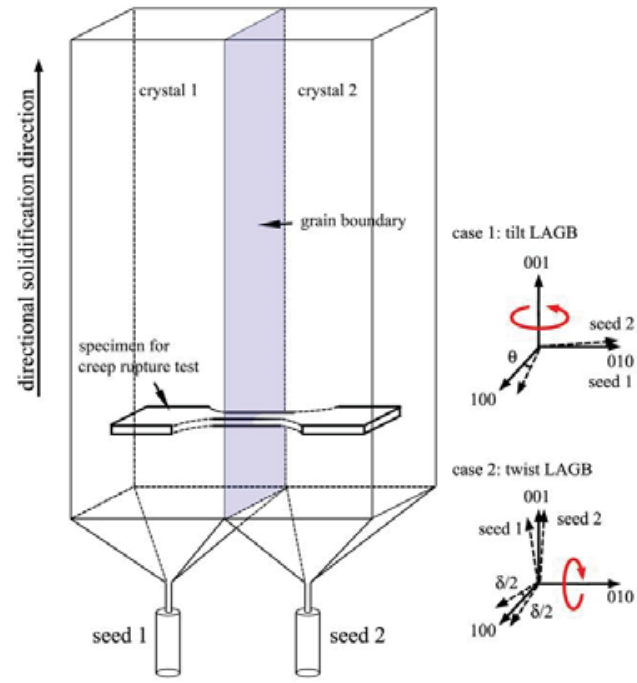

Figure 1. Schematic of bicrystal slab casting prepared by the seed crystal method and dog-bone shape creep specimen extracted from the bicrystal.

The angles of LAGBs were determined by scanning electron microscopy (SEM, S-3400N) with electron backscatter diffraction (EBSD). The LAGBs were characterized by optical microscopy (OM, Axiovert200MAT), SEM and high resolution electron microscopy (HR-TEM, FEI Tecnai G2 F30). Dog-bone shape creep rupture specimens were cut and machined from the castings, as shown in Figure 1, the gauge section dimension was $15 \mathrm{~mm}$ (length) $\times 5 \mathrm{~mm}$ (width) $\times 2 \mathrm{~mm}$ (thickness). Creep rupture tests were performed in ambient atmosphere at $760{ }^{\circ} \mathrm{C}$, and the creep loading axis was perpendicular to the LAGBs. Some of the creep tests were interrupted for microstructure observation. SEM was also used to observe the microstructural damage in the vicinity of LAGB. TEM (FEI Tecnai G2 F20) was used to analyze and identify the dislocation configuration and its interaction with LAGBs.

\section{Results}

\section{Morphology of LAGBs}

The bicrystal slabs with near-tilt and near-twist LAGBs were successfully prepared, and Figure 2 shows the typical microstructure of a grain boundary around $15 \mathrm{deg}$. Two crystals with 14.9 deg misorientation of the secondary dendritic arms on (001) plane on each side of the tilt boundary can be observed in Figure $2 \mathrm{a}$ and $\mathrm{b}$. A twist boundary was obtained with the $14.4 \mathrm{deg}$ misorientation of primary dendritic crystals on (010) plane, as shown in Figure $2 \mathrm{~d}$ and e. Figure $2 \mathrm{c}$ and $\mathrm{f}$ show the schematic arrangement of the dendrites. It is difficult to identify the tilt boundary along the DS direction, and the twist boundary along or perpendicular to the DS direction by morphological observation at low magnification. However, it was worthy to note that, the misorientation of dendritic crystals at other sides of the boundary

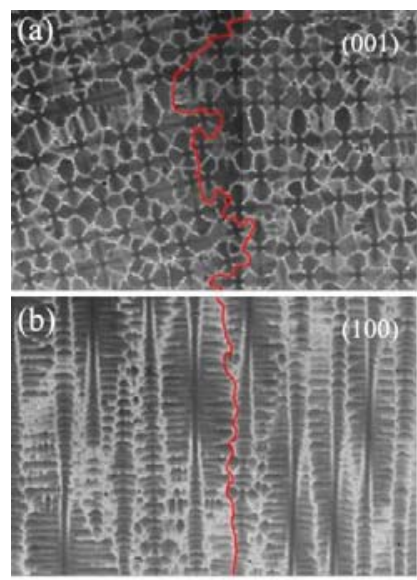

(c)
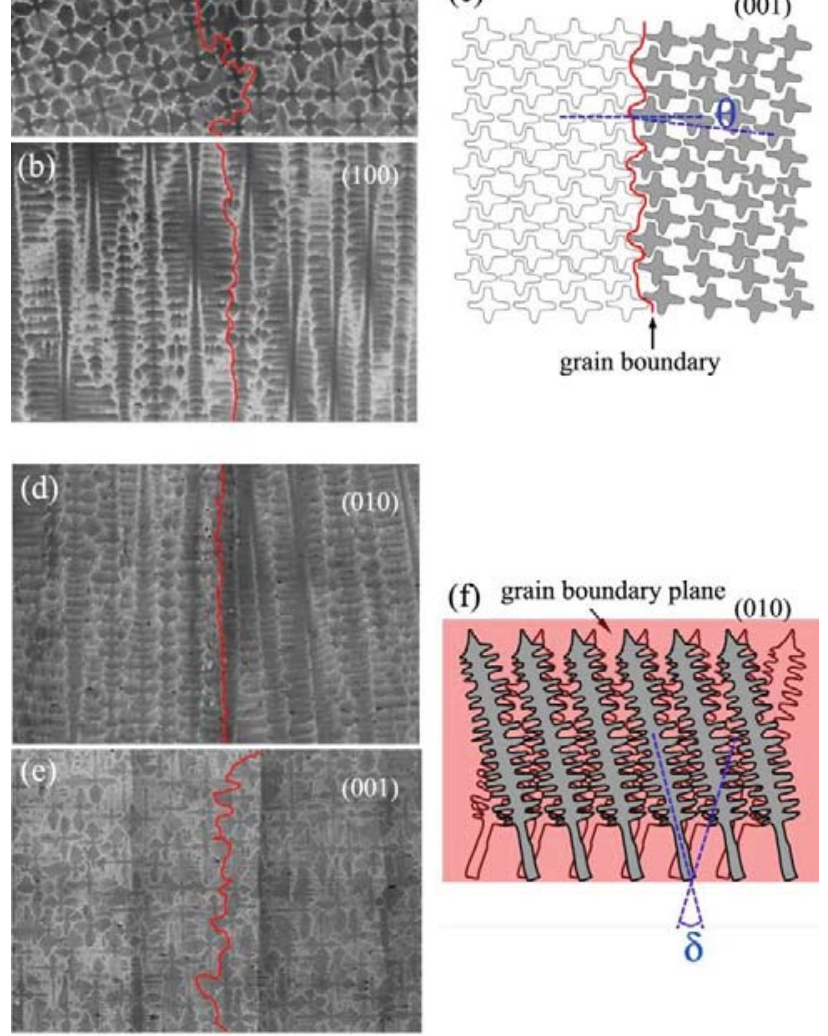

(f) grain boundary plane

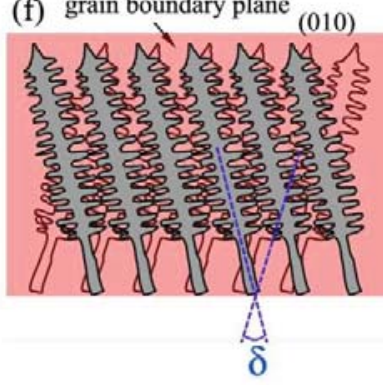

Figure 2. Microstructure observation of (a), (b), (c) tilt boundary $(\theta=14.9 \mathrm{deg})$, and (d), (e), (f) twist boundary ( $\delta=14.4 \mathrm{deg})$ in DD10 SX superalloy. (a) and (e) dendrites morphology perpendicular to the DS direction; (b) and (d) dendrites morphology along the DS direction; (c) and (f) schematic of dendrites morphology.

(i.e., (010) and (100) for tilt, and (001) and (100) for twist) were restricted as low as possible during the bicrystal growth. EBSD results indicate, for example, misorientation at the other sides of the $15 \mathrm{deg}$ boundary were $<2 \mathrm{deg}$ in these samples.

The SEM microstructure of the grain boundaries after heat treatment is shown in Figure 3. The morphology of the two kinds of grain boundaries was very similar. At (001) plane of tilt

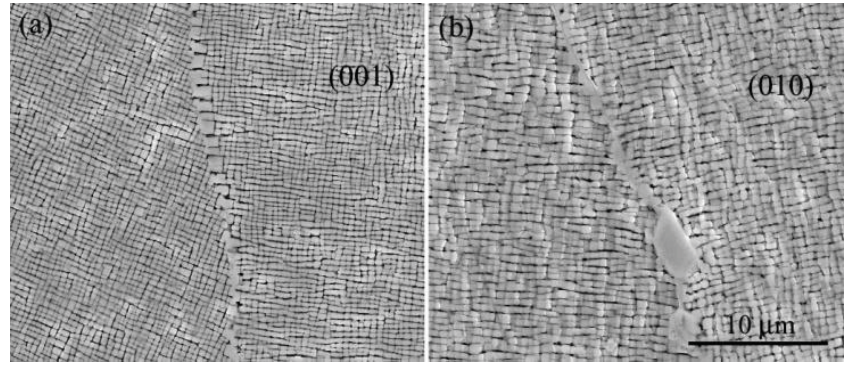

Figure 3. Microstructure of (a) tilt boundary and (b) twist boundary in DD10 SX superalloy after heat treatment. 

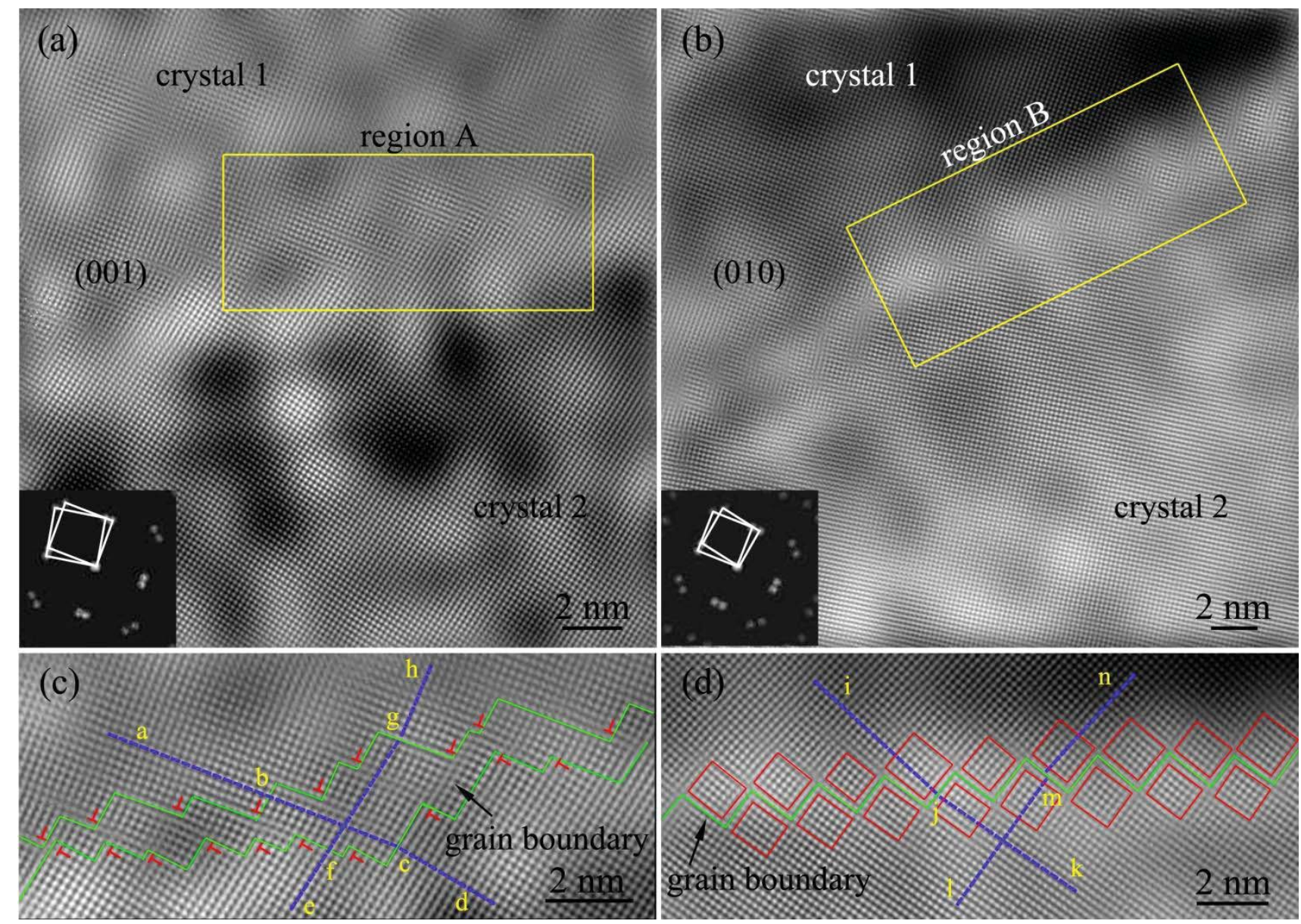

Figure 4. HR-TEM observation of (a) tilt boundary ((c) is the high magnification of region A in (a)) and (b) twist boundary ((d) is the high magnification of region B in (b)) in DD10 SX superalloy after heat treatment. The boundaries have a misorientation around 15 deg. There was an obvious distortion in the regions A and B. Extra half an atomic planes can be found in region A, and no extra half an atomic planes can be noticed from the red squares. The atom plane (line ab) in one crystal extends into transition region, and then deflection takes place at point $\mathrm{c}$ induced by an edge dislocation. The atom plane (line ef) shows similar configuration, as shown in (c). In (d), the atom planes (line $\mathrm{ij}$ and $\mathrm{lm}$ ) extend into the neighbouring crystals and the deflections occur at point $\mathrm{j}$ and $\mathrm{m}$ effected by the screw dislocations, respectively, resulting in the misorientation of crystals.

boundary or (010) plane of twist boundary, the orientation of the $\gamma^{\prime}$ phase in one grain was different from that in the other grain. In addition, there were obviously large $\gamma^{\prime}$ phases at the boundaries.

TEM was used to further characterize the microstructure of the LAGBs. For tilt boundaries, the normals of neighboring crystal face near the boundary from observation plane were parallel to each other. HR-TEM observation was relatively easy to perform. Figure 4a shows the HR-TEM image of a tilt boundary with misorientation $\sim 15 \mathrm{deg}$, and high magnification of region $\mathrm{A}$ is visible in Figure 4c. It was found that the tilt boundary was composed of an uninterrupted transition region. Its bilateral borders displayed the irregular bench feature. An edge dislocation inserted into each concave corner of the bench borders matching the neighboring atomic planes of respective grain crystal, causing the misorientation at grain boundary. For twist boundaries, it was difficult to see clearly the HR-TEM image of twist boundary due to the misorientation of the normals of neighboring crystal face near boundary. Considering twist boundary with a misorientation around $15 \mathrm{deg}$, the orientation of two grains deviates symmetrically about $7 \mathrm{deg}$ from [001]. A compromise orientation

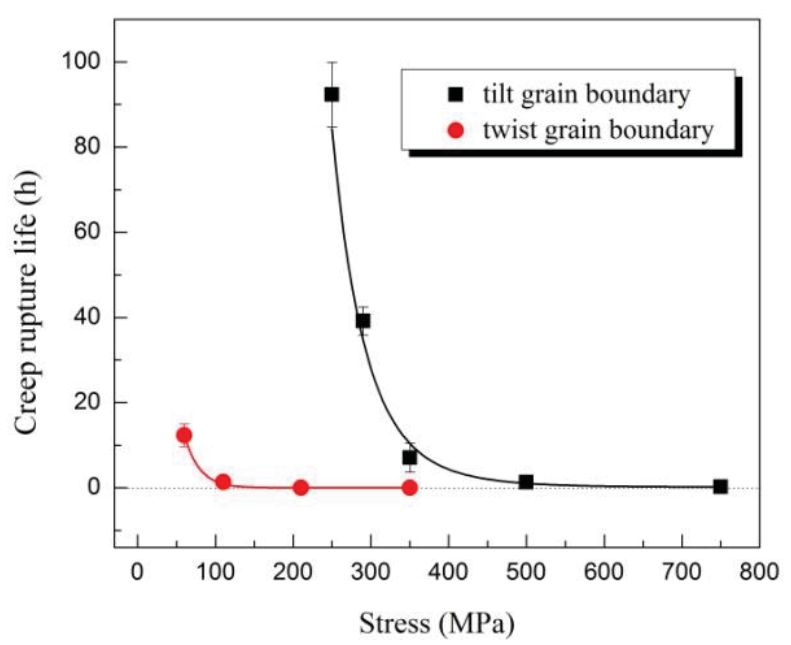

Figure 5. Creep rupture life of the DD10 alloy with transverse LAGB at $760{ }^{\circ} \mathrm{C}$, showing the strong correlation between creep rupture life and the type of the boundary. 
with the incident beam parallel to [001] direction was adopted to image the microstructures near the boundary. Compared to the tilt boundary, the dislocation array for the twist boundary was composed of a row of stair-stepping atomic arrangements, and the misorientation was induced by two sets of orthogonal screw dislocations, as seen in Figure 4d (which is the high magnification of region B from Figure 4b).

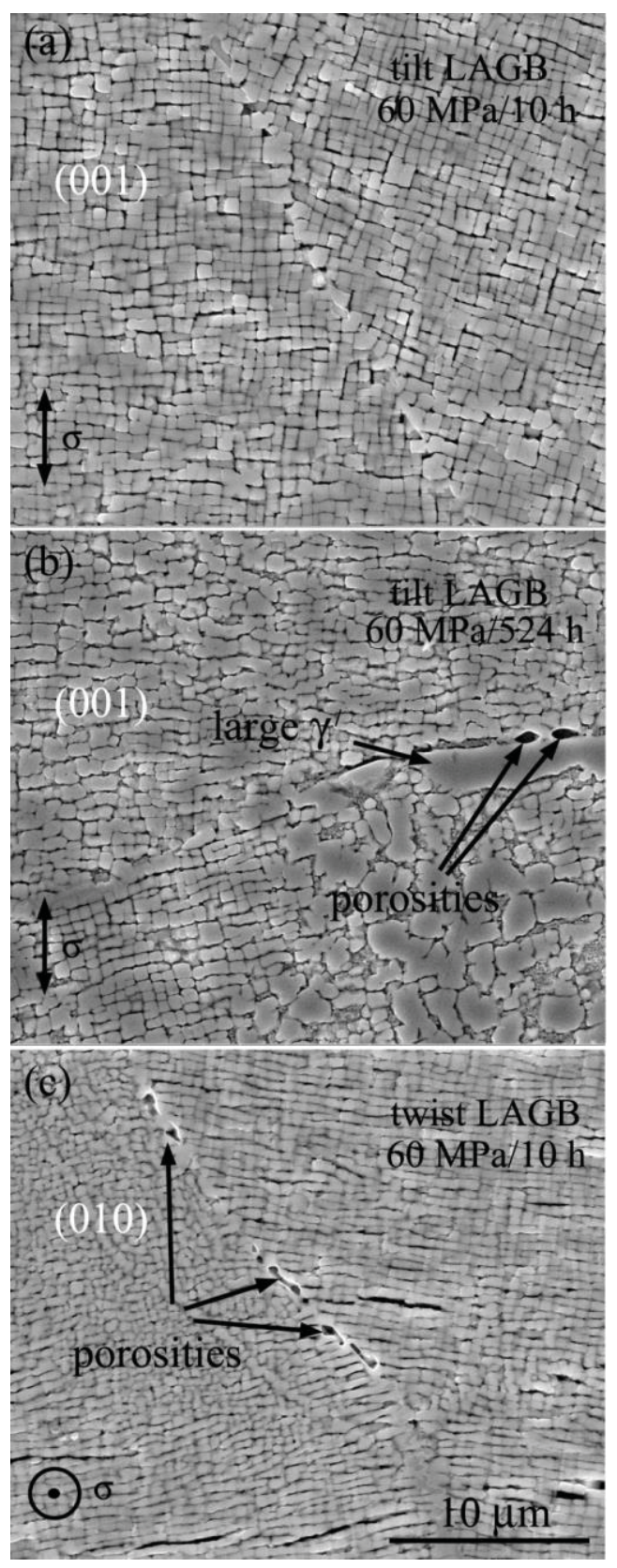

Figure 6. Microstructure morphologies near the grain boundaries of rupture interrupted specimens at $760^{\circ} \mathrm{C} / 60 \mathrm{MPa}$. (a) and (b) tilt boundary interrupted at $10 \mathrm{~h}$ and $524 \mathrm{~h}$, respectively; (c) twist boundary interrupted at $10 \mathrm{~h}$.

\section{$\underline{\text { Creep rupture properties }}$}

The creep rupture life as a function of the applied stress on the specimens with a grain boundary around $15 \mathrm{deg}$ are plotted in
Figure 5. It was apparent that the creep rupture life at $760{ }^{\circ} \mathrm{C}$ decreased with the increasing in applied stress for both tilt and twist specimens. Clearly, the specimen with a twist boundary of 14.4 deg can barely endure plastic deformation even at $60 \mathrm{MPa}$, and the fracture occurred readily along the grain boundary. In contrast, the specimen with a tilt boundary (14.9 deg) exhibited much better compatibility even at high applied stress. The same trend was also found in other specimens with $5 \mathrm{deg}$ and $10 \mathrm{deg}$ grain boundary misorientation. For example, creep life of specimen with a tilt boundary of $4.8 \mathrm{deg}$ at $760{ }^{\circ} \mathrm{C} / 550 \mathrm{MPa}$ was $173 \mathrm{~h}$. But creep life of specimen with a twist boundary of $6.7^{\circ}$ at $760{ }^{\circ} \mathrm{C} / 550 \mathrm{MPa}$ was only $83 \mathrm{~h}$. Therefore, the present results demonstrate that the intermediate-temperature creep rupture behavior was strongly correlated to the grain boundary types.

\section{Creep damage behavior}

In this study, all tested bicrystal creep specimens failed along their grain boundaries. In order to reveal the creep damage behavior of bicrystal alloy, interrupted creep tests were performed to investigate the evolution of the microstructure and damage during creep. Typical damage microstructure near the grain boundary of the interrupted specimens is given in Figure 6. It can be seen that there was no obvious damage to the specimen at the tilt boundary after $10 \mathrm{~h}$ of creep deformation at $760^{\circ} \mathrm{C} / 60 \mathrm{MPa}$ (Figure 6a), and only few holes emerged at large $\gamma^{\prime}$ phases as the deformation time extended to $524 \mathrm{~h}$ (Figure 6b). However, a large number of holes were clearly visible for the specimen even though the interrupted life was $10 \mathrm{~h}$ in the specimen containing a twist boundary (Figure $6 c)$.
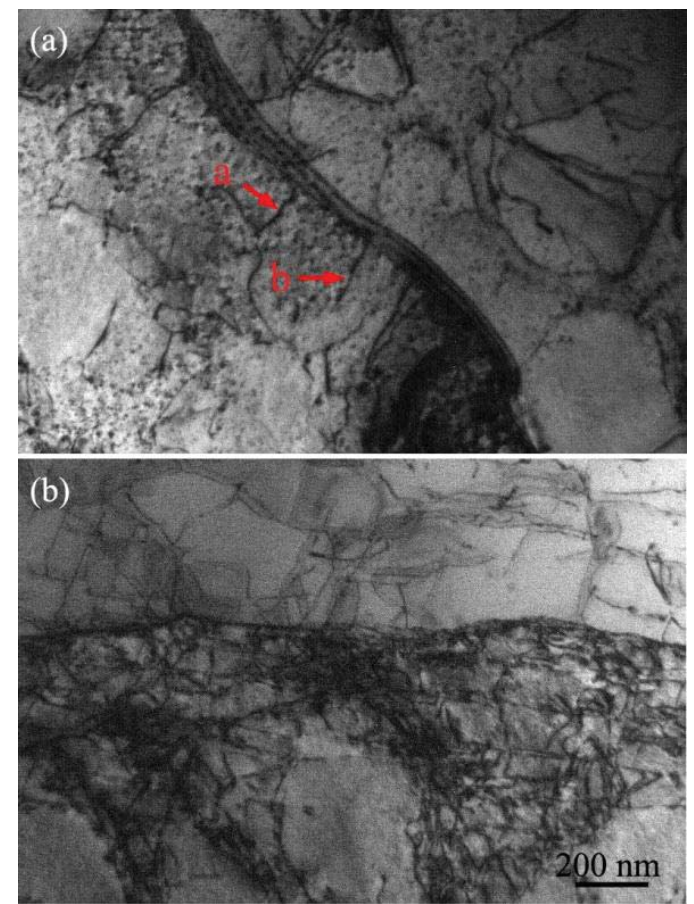

Figure 7. Dislocation configurations on (001) plane near the (a) tilt boundary; (b) twist boundary of bicrystal interrupted at 760 ${ }^{\circ} \mathrm{C} / 60 \mathrm{MPa}$ for $10 \mathrm{~h}$. It was difficult to observe the grain boundary dislocation density under two beam conditions due to the misorientation of two grains. A concessional attempt with the incident beam parallel to [001] direction was thus adopted to obtain the image. 
In order to further clarify the role of different type grain boundaries on intermediate-temperature creep deformation, dislocation configurations of the bicrystal specimens containing tilt and twist boundary after $10 \mathrm{~h}$ creep deformation were also observed. Low dislocation density on both sides of the tilt boundary (Figure 7a) can be observed, indicating that the dislocation (for example "a" and "b") can pass through the grain boundary during creep. On the contrary, the dislocation density is very high on one side of twist boundary (Figure $7 b$ ), which means that most dislocations accumulate at grain boundary instead of passing through it.

\section{Discussion}

Generally, the rupture life of specimens with the transverse LAGB is related to the two dominant factors as the type and angle of grain boundary which can directly affect the interaction between LAGB and mobile dislocation. In the present experiments when focusing primarily on the behavior of a boundary with misorientation around $15^{\circ}$, there should be no significant difference in the effect of the angles of LAGBs on the rupture life of the specimens due to the similar misorientation for both boundary types. Therefore, the intrinsic dislocation configuration

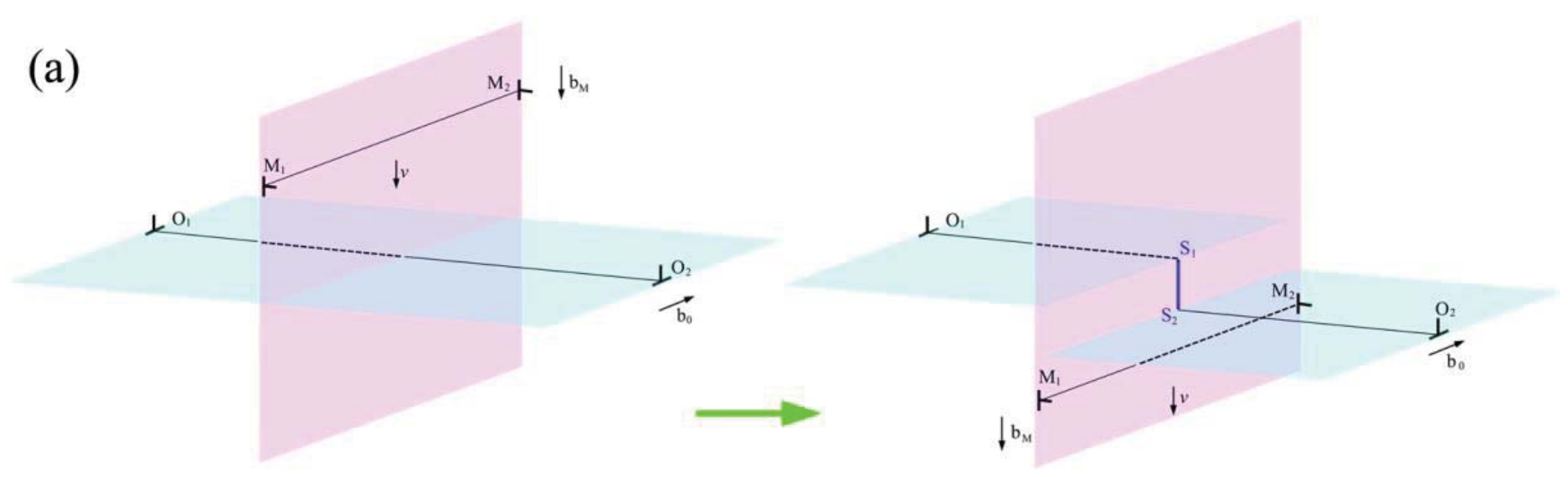

(b)

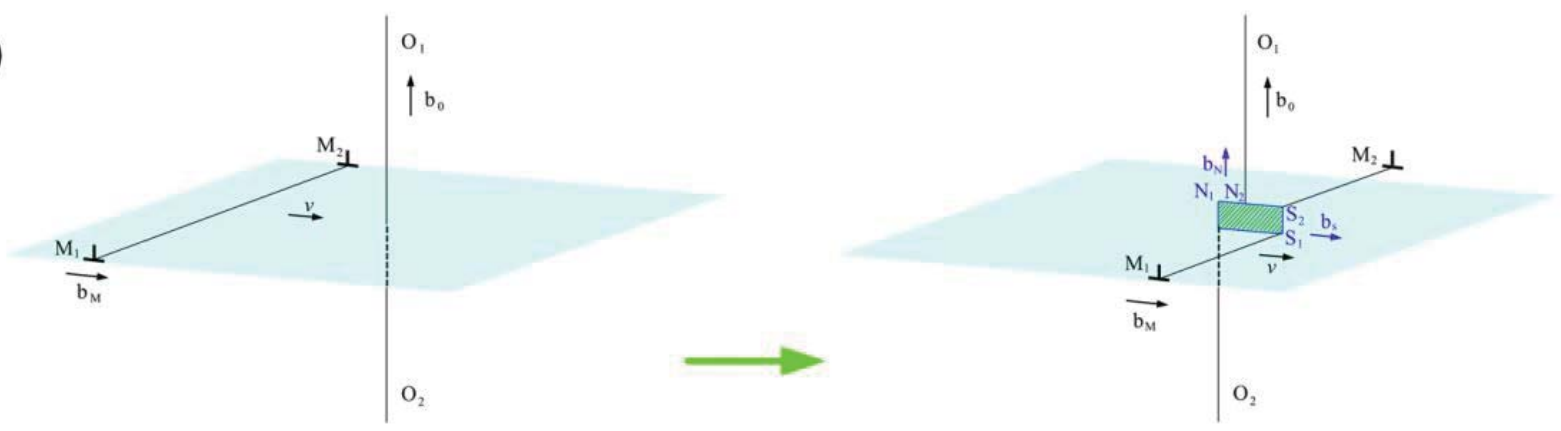

(c)

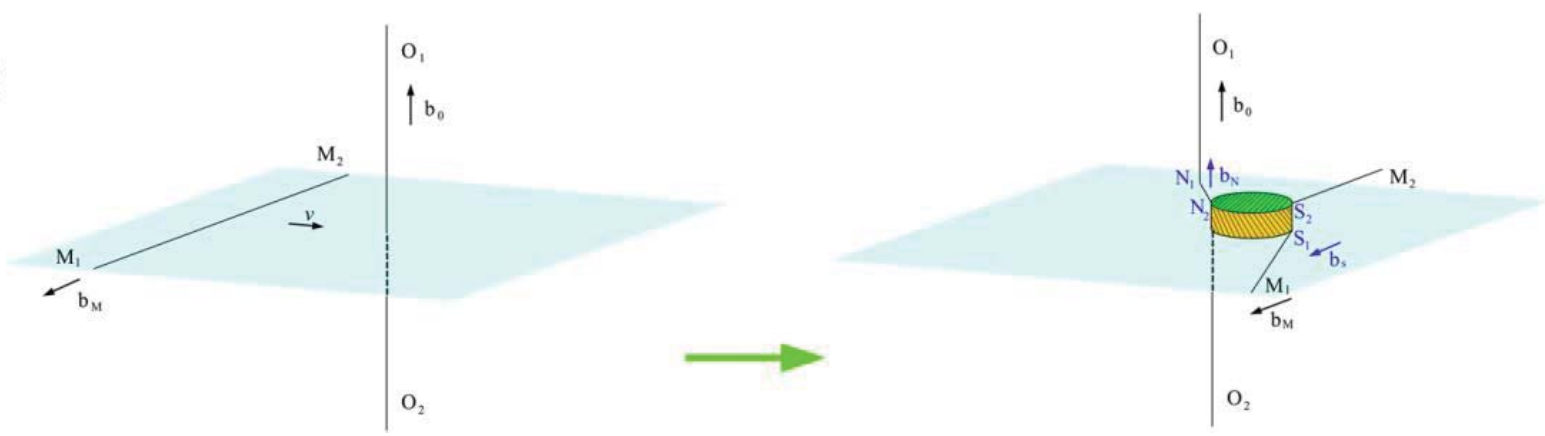

Figure 8. Schematic of the interaction between a mobile dislocation and tilt $(\mathrm{a}, \mathrm{b})$ or twist $(\mathrm{b}, \mathrm{c})$ boundary. (a) edge dislocation and edge dislocation, (b) edge dislocation and screw dislocation (Formation of jog only at edge dislocation), and (c) screw dislocation and screw dislocation during creep deformation (Formation of jog at both screw dislocations) 
at the grain boundaries (i.e., edge or screw dislocation) plays a key role during the intermediate-temperature creep deformation.

All of the dislocations formed during deformation can be regarded as being composed of an edge dislocation section and a screw dislocation section. As the mobile dislocation encountering the tilt boundary containing edge dislocation, there are mainly edge-edge dislocation interaction and edge-screw dislocation interaction. No matter the mobile dislocations are edge or screw, they can all pass through the tilt boundary and keep sliding, as schematically shown in Figure $8 \mathrm{a}$ and $\mathrm{b}$. Similar results showing the dislocations passing through tilt LAGB have also been reported by Zhang et al $[7,8]$. On the other hand, as a mobile dislocation encountering twist LAGB, there are mainly edge-screw and screw-screw dislocation interaction at LAGB. According to Figure $8 \mathrm{~b}$ and c, it is easy to form jog at mobile dislocation. Since the slip plane of jog is different from that of the original mobile dislocation, jog may be an obstacle for further movement of the dislocation, resulting in severe dislocation tangle at twist LAGB and rapid fracture of the specimen.

\section{Summary}

Our present results may show some impacts on the casting industry since LAGB is one of the most common defects in the SX practice. The results can be summarized as:

1. The near-tilt boundary in SX alloys shows a transition region, and is composed of intrinsic edge dislocations. However, twist boundary shows almost no transition region, and is mainly made up of intrinsic screw dislocations.

2. The tilt boundary shows a much better intermediatetemperature creep resistance compared to that of a twist boundary.

3. It seems that the mobile dislocations can pass through the tilt boundary, but they may pile up in ahead of the twist boundary due to the different dislocation interaction at the grain boundaries. This may explain the significant variation of the creep rupture behavior in specimens containing different types of grain boundaries.

\section{Acknowledgement}

This work was financially supported by the National High Technology Research and Development Program of China (863 Program) under grant No. 2012AA03A513 and No. 2012AA03A511 and the National Natural Science Foundation of China under grant No. 51171193. The authors are grateful for these supports.

\section{References}

1. J.W. Aveson, G. Reinhart, H. Nguyen-Thi, N. MangelinckNoël, A. Tandjaoui, B. Billia, K. Goodwin, T.A. Lafford, J. Baruchel, H.J. Stone and N.D'Souza, "Dendrite bending during directional solidification”, Superalloys 2012, ed. E.S. Huron, R.C. Reed, M.C. Hardy, M.J. Mills, R.E. Montero, P.D. Portella and J. Telesman, (Pennsylvania, PA: The Minerals, Metals and Materials Society, 2012), 615-624.
2. J.W. Aveson, P.A. Tennant, B.J. Foss, B.A. Shollock, H.J. Stone and N.D'Souza, "On the origin of sliver defects in single crystal investment castings", Acta Materialia, 61 (2013), 51625171.

3. Q.Z. Chen, C.N. Jones and D.M. Knowles, "The grain boundary microstructures of the base and modified RR 2072 bicrystal superalloys and their effects on the creep properties", Materials Science Engineering A, 385 (2004), 402-418.

4. J.C. Stinville, K. Gallup and T.M. Pollock, "Transverse creep of nickel-base superalloy bicrystals", Metallurgical Materials Transactions A, 46(2015), 2516-2529.

5. J.Q. Zhao, J.R. Li, S.Z. Liu, H.L. Yuan and M. Han, "Effects of low angle grain boundaries on the stress rupture properties of single crystal superalloy DD6". Journal of Aeronautical Materials, 27 (2007), 6-10, (in Chinese).

6. A. Morawiec, "On the frequency of occurrence of tilt and twist grain boundaries”, Scripta Materialia, 61 (2009), 438-440.

7. P. Zhang, Z.J. Zhang, L.L. Li and Z.F. Zhang, "Twin boundary: stronger or weaker interface to resist fatigue cracking?", Scripta Materialia, 66 (2012), 854-859.

8. Z.F. Zhang and Z.G. Wang, "Effect of grain boundaries on cyclic deformation behavior of copper bicrystals and columnar crystals", Acta Materialia, 46 (1998), 5063-5072. 